\section{Niveles séricos del antígeno prostático específico en varones expuestos y no expuestos al arsénico en el agua de bebida en Tucumán, Argentina.}

\author{
ANALÍA SORIA DE GONZÁLEZ ${ }^{1, \mathrm{a}}$, LILIANA TEFAHA, \\ ROSA GUBER $^{1, \mathrm{~b}}$, NILDA ARIAS $^{1, \mathrm{~b}}$, CLAUDIO ROMERO $^{2}$, \\ MATEO MARTÍNEZ ${ }^{2, c}$, MARTA VALDIVIA $^{1, \mathrm{~b}}$, NOEMI SANDOVAL $^{1, \mathrm{~b}}$, \\ ROXANA TOLEDO ${ }^{2, c}$, MARÍA CZEJACK ${ }^{2, c}$
}

\section{Total prostatic specific antigen levels among subjects exposed and not exposed to arsenic in drinking water}

Background: There is an association between arsenic exposure and prostatic cancer mortality. Aim: To analyze and to compare the serum levels of total prostatic specific antigen (TPSA) in asymptomatic men of a rural zone, exposed and not exposed to high concentrations of arsenic (As) in drinking water. Material and Methods: The study included 161 men that were subjected to a clinical evaluation, serum TPSA determination and a trans rectal ultrasonography. Exposed and non-exposed subjects were divided in groups GA, GB and GC according to TPSA levels $(<4 \mathrm{ng} / \mathrm{ml}, 4.1-10$ $\mathrm{ng} / \mathrm{ml}$ and $>10.1 \mathrm{ng} / \mathrm{ml}$ respectively). Results: Exposed and non-exposed subjects had similar TPSA levels. Among exposed subjects, 88.2, 8,8 and 3\% were ascribed to groups GA, GB and GC respectively. The figures for non-exposed subjects were 90. $6,6,3$ and 3,1\%. The area under the receiver operating characteristic (ROC) curve for TPSA in exposed and non-exposed subjects was 0.64 (95\% confidence intervals (CI) 0.40-0.9) and 0.71 (95\% CI 0.6-0.8) respectively. Conclusions: No differences in TPSA levels between subjects exposed and non-exposed to arsenic were observed in this study.

(Rev Med Chile 2011; 139: 1581-1587).

Key words: Antigens, neoplasm; Arsenic; Prostatic neoplasms.

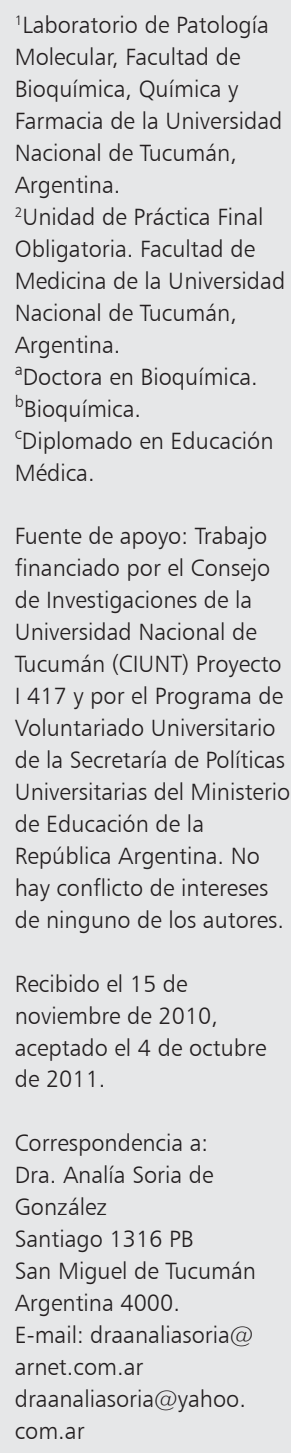

E 1 cáncer de próstata $(\mathrm{CP})$ es el cáncer más comúnmente diagnosticado en el sexo masculino y después del de pulmón, es la segunda causa de muerte por cáncer. Su incidencia y mortalidad continúan en aumento, conformando un importante problema de salud pública, en estrecha relación con el aumento de la longevidad poblacional ${ }^{1}$. Hay factores de riesgo involucrados en su desarrollo, siendo los más importantes la edad (es una patología de hombres mayores), la etnia y la historia familiar ${ }^{2}$. El antígeno prostático específico total (APET) es un marcador tumoral útil para la evaluación del paciente con $\mathrm{CP}$ en los distintos estadíos de la enfermedad. La determinación sérica de este marcador es el responsable del aumento de la incidencia de este cáncer en las últimas dos décadas ${ }^{3}$. La utilidad y aplicación del APET en los programas de detección precoz ha sido cuestionada debido a que, si bien se podría reducir las muertes por $\mathrm{CP}$, se debe considerar los riesgos que se generan por la detección temprana y los tratamientos que reciben los hombres quienes podrían tener un cáncer indolente que no representa una amenaza de vida para el paciente ${ }^{4}$. 
Numerosos trabajos han documentado la falta de especificidad del APET, ya que se encontraron aumentos de este marcador en patologías benignas, como hiperplasia prostática benigna (HPB) y prostatitis ${ }^{5}$. La Sociedad Americana del Cáncer recomienda que los hombres asintomáticos, quienes tienen una expectativa de vida mayor a 10 años, debieran tener la oportunidad de participar en los programas de detección precoz, bajo consentimiento informado, siempre que hayan recibido la información sobre las incertidumbres, los riesgos y los beneficios asociados ${ }^{6}$. El 25-35\% de los hombres con niveles moderadamente elevados de APET (4-10 ng/mL) a los que se les realizaron biopsias, se les detectó la presencia de CP. Cuando los niveles de APET son mayores a 10 $\mathrm{ng} / \mathrm{mL}$ la especificidad del marcador es mayor a $50 \%{ }^{7}$. El APET en circulación corresponde aproximadamente a la suma del APE acomplejado y el libre. La principal aplicación clínica del APE libre (APEL) es la reducción de biopsias innecesarias en hombres con niveles de APET entre 4 y $10 \mathrm{ng} / \mathrm{mL}$. Asimismo, se recomienda la determinación del APEL para diferenciar HPB de CP en individuos con niveles menores a $10 \mathrm{ng} / \mathrm{mL}$ y EDR negativo ${ }^{8,9}$. Existen otros factores que estarían involucrados en la carcinogénesis del CP. Particularmente, fue documentada la relación entre la exposición al arsénico (As) y el aumento de la tasa de mortalidad por CP en países con esta problemática, como China, India, Estados Unidos de Norteamérica (USA), Taiwan ${ }^{10,11}$. En el este de la provincia de Tucumán, el arsenicismo crónico configura un grave problema de salud pública y de alta importancia social, que incide en comunidades pobres y con un nivel de instrucción limitado. El As llega al organismo a través de agua contaminada con concentraciones elevadas de este metaloide, lo que implica que grandes grupos poblacionales pueden ser afectados por sus efectos nocivos, a medida que se acumula en el organismo ${ }^{12}$. En relación a su letalidad potencial, la Agencia de Protección Ambiental de los Estados Unidos, clasifica al As como carcinógeno del grupo $\mathrm{A}^{13} \mathrm{y}$ el Centro de Investigaciones sobre Cáncer lo incluye en el grupo I, indicando en ambos casos que el agente es carcinógeno para los seres humanos ${ }^{14}$. Estudios experimentales realizados en líneas celulares de células epiteliales prostáticas humanas que fueron expuestas al As, sufrieron transformación maligna demostrando que el As en estas condiciones, in- duce la carcinogénesis ${ }^{15}$. La mortalidad por cáncer broncopulmonar, vejiga urinaria, vías urinarias, riñón, hepático y piel aumenta por la exposición crónica a As, la cual no es modificada por exposición prenatal o perinatal a este elemento ${ }^{16,17}$. El objetivo del presente trabajo fue analizar y comparar los niveles séricos del APET en hombres residentes en una zona rural, asintomáticos, expuestos y no expuestos a concentraciones elevadas de arsénico en el agua de consumo.

\section{Pacientes y Métodos}

\section{Población estudiada}

Este trabajo corresponde a un estudio descriptivo de corte transversal, de carácter exploratorio. Fue realizado entre los años 2005 y 2009 en una población rural en el departamento de Graneros de la provincia de Tucumán, Argentina, en donde la Universidad Nacional de Tucumán (UNT) posee unidades docente asistenciales permanentes denominadas Pasantías Rurales, pertenecientes a la Facultad de Medicina. Se realizaron entrevistas individuales confeccionadas ad-hoc, con datos demográficos y urológicos, a 221 varones mayores de 50 años que habitaban en forma permanente por más de 10 años, previo consentimiento por escrito, explicándoles la finalidad de este estudio y las controversias relacionadas con la determinación del APET en la detección precoz del CP. Como criterios de exclusión se tuvieron en cuenta: negativa a la entrevista, al examen físico o a la extracción sanguínea, diagnóstico previo de $\mathrm{CP}$ o cualquier condición que alterara la determinación del APET, tales como infección o inflamación de la próstata, tacto rectal u otros estudios que involucren manipulación prostática en la semana previa a la extracción de sangre. Se seleccionaron 161 hombres asintomáticos, los cuales fueron citados a los lugares de referencia con una muestra de agua de pozo domiciliario, recolectada en frascos de plástico limpios, las cuales fueron remitidas al Laboratorio de Patología Molecular de la Facultad de Bioquímica, Química y Farmacia de la UNT, en donde se cuantificó la concentración de As. A todos los pacientes bajo estudio, se les realizó historia clínica completa y determinación del APET y APE libre. El estudio se completó con la ecografía vesico-prostática pre y post miccional y en los casos que lo requirieron se realizó biopsia ecodirigida transrectal. 


\section{Recolección de muestras y métodos de laboratorio}

La determinación de As fue realizada según el método cuantitativo modificado de Gutzei, en donde el elemento As forma hidruro de arsénico que reacciona con el dietilditiocarbamato de plata para dar un compuesto rojizo, cuya intensidad de color, medida fotométricamente a $530 \mathrm{~nm}$, es proporcional a la concentración de As presente en la muestra de agua (GT lab). Las muestras de sangre venosa fueron colectadas en tubos libres de anticoagulantes y dejadas coagular espontáneamente 30 minutos a $37^{\circ} \mathrm{C}$, separándose el suero por centrifugación, conservado a $-20^{\circ} \mathrm{C}$ hasta su uso. La determinación sérica del APET y APEL consiste en un análisis cuantitativo automatizado con metodología ELFA (Enzyme-Linked Fluorescent Assay) en un analizador MINIVIDAS de Laboratorio Biomerieux. Los valores de referencia de normalidad son entre 0 y $4,0 \mathrm{ng} / \mathrm{ml}$.

\section{Análisis Estadístico}

Se realizó un análisis descriptivo de las variables. El contraste de normalidad se realizó con el test de Shapiro-Wilks. Para el análisis estadístico utilizamos distribuciones de frecuencia, media o mediana dependiendo del tipo de distribución: paramétrica mediante el test $t$ de Student o no paramétrica con el test Mann-Whitney U. Los valores de corte del APET en hombres expuestos y no expuestos a niveles elevados al As en el agua de consumo se determinaron mediante las correspondientes curvas ROC y su área bajo la curva (ABC). El análisis estadístico se efectuó mediante el programa Statistical Package for Social Sciences (SPSS 17.0) para Windows. Se estableció una asociación estadísticamente significativa cuando se halló un valor de $\mathrm{p}<0,05$.

\section{Resultados}

Se analizaron los niveles de As en las muestras de agua de consumo de la población estudiada de las cuales $34(21,1 \%)$ tenían valores de As mayores a $0,01 \mathrm{mg} / \mathrm{L}$ y $127(78,9 \%)$ tenían niveles permitidos para el consumo. El valor promedio \pm desviación estándar del As dosado del Grupo Expuesto (GEx) fue de 0,155 $\pm 0,170 \mathrm{mg} / \mathrm{L}$. La concentración máxima medida fue de $0,820 \mathrm{mg} / \mathrm{L}$. La edad promedio de los hombres del GEx y Grupo No Expuesto (GNoEx) fue de 59,1 \pm 7,1 y 61,4 \pm 8,9 años, respectivamente. Ambos grupos fueron divididos en GA, GB y GC según si los niveles del APET fueron menores o iguales a $4 \mathrm{ng} / \mathrm{mL}$, entre 4,1 y $10 \mathrm{ng} / \mathrm{mL}$ o mayor a $10,1 \mathrm{ng} / \mathrm{mL}$. El GA fue subdividido en GA1 y GA2. El GA1 correspondió a varones sin patologías prostáticas y el GA2 a varones con niveles normales de APET a los que les fueron diagnosticados patologías prostáticas benignas. En el GEx el 70,6\% pertenecía al grupo GA $1 ; 17,6 \%$ al GA $2 ; 8,8 \%$ al GB y $3,0 \%$ al GC. En el GNoEx 78,0\% pertenecían a los GA 1; 12,6\% al GA 2; 6,3\% al GB y 3,1\% al GC. Cuando se analizaron los niveles del APET en el GAEx y GANoEx no se encontraron diferencias significativas entre los que tenían o no patologías prostáticas. Cuando se evalúa el GAEx y el GANoEx completo se encontraron diferencias estadísticamente significativas entre ambos grupos $(\mathrm{p}<0,03)$ (Tabla 1). En la Figura 1 puede observarse el diagrama de caja correspondiente. No se encontraron diferencias estadísticamente significativas entre los niveles del APET en el GBNoEx y GBEx (Tabla 1). No se comparó el GC ya que hay un solo paciente expuesto que pertenece a este grupo (Tabla 1). Para determinar el mejor punto de corte del APET en hombres expuestos y no expuestos a niveles elevados al As en el agua de consumo se estimó el área bajo la curva ROC (Tabla 2). El área bajo la curva del APET en GNoEx fue de 0,71 IC 95\% $(0,58-0,84)$ (Figura 2). El área obtenida bajo la curva del APET en GEx fue de 0,64 IC 95\% (0,400,88 ) (Figura 3 ).

\section{Tabla 1. Valores medios \pm desviación estándar de APET $(\mathrm{ng} / \mathrm{mL})$ en varones que consumen (expuestos) y no consumen (no expuestos) agua contaminada con As}

\begin{tabular}{|cccrc|}
\hline & N & Expuestos & N & No expuestos \\
GA & 30 & $0,91 \pm 0,55^{*}$ & 115 & $1,14 \pm 0,82^{*}$ \\
GB & 3 & $6,43 \pm 1,37 * *$ & 9 & $6,59 \pm 1,69 * *$ \\
GC & 1 & 14,5 & 3 & $23,2 \pm 2,41$ \\
\hline
\end{tabular}

${ }^{*}(\mathrm{p}<0,03) .{ }^{* *}$ (no significativo). GA: varones con APET sérico $<$ o igual a $4 \mathrm{ng} / \mathrm{mL}$. GB: varones con APET sérico entre 4,1 y $10 \mathrm{ng} / \mathrm{mL}$. GC: varones con APET sérico > a 10,1 ng/mL. $\mathrm{N}$ : número de personas estudiadas. 


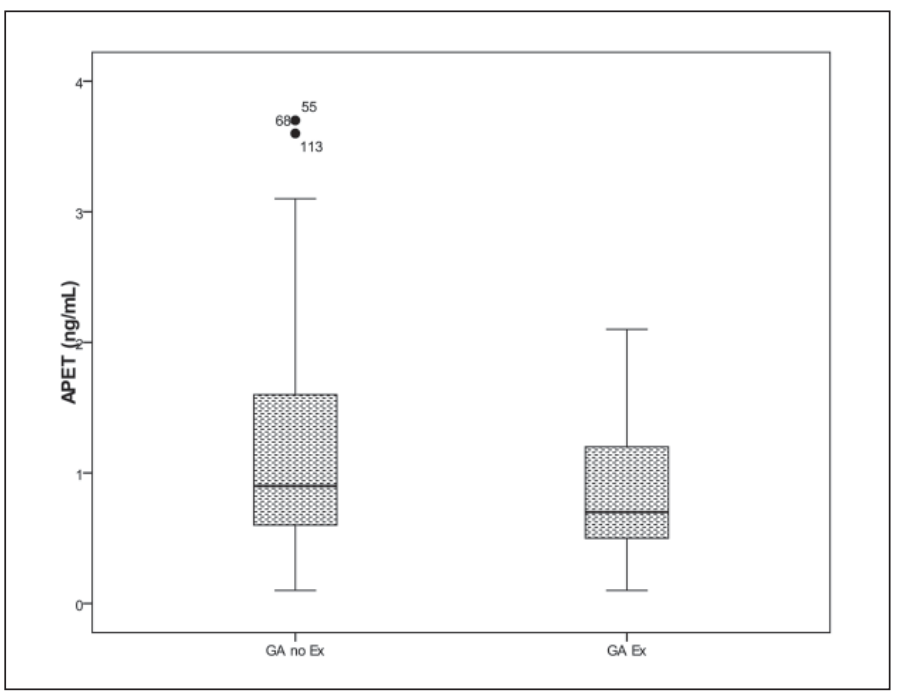

Figura 1. Niveles Séricos del APET en el GA No Ex y Ex a concentraciones elevadas de As en el agua de bebida.

Tabla 2. Sensibilidad y especificidad para distintos puntos de corte de APET en varones que consumen (expuestos) y no consumen (no expuestos) agua contaminada con As

\begin{tabular}{|cccccc|}
\hline & Expuestos & & \multicolumn{3}{c|}{ No expuestos } \\
\hline PC (ng/mL) & S (\%) & E (\%) & PC (ng/mL) & S (\%) & E (\%) \\
$>1,40$ & 50 & 79 & $>1,35$ & 61 & 71 \\
$>1,93$ & 40 & 92 & $>2,35$ & 50 & 90 \\
$>2,80$ & 40 & 100 & $>4,05$ & 43 & 100 \\
\hline
\end{tabular}

PC: punto de corte; S: sensibilidad; E: especificidad.

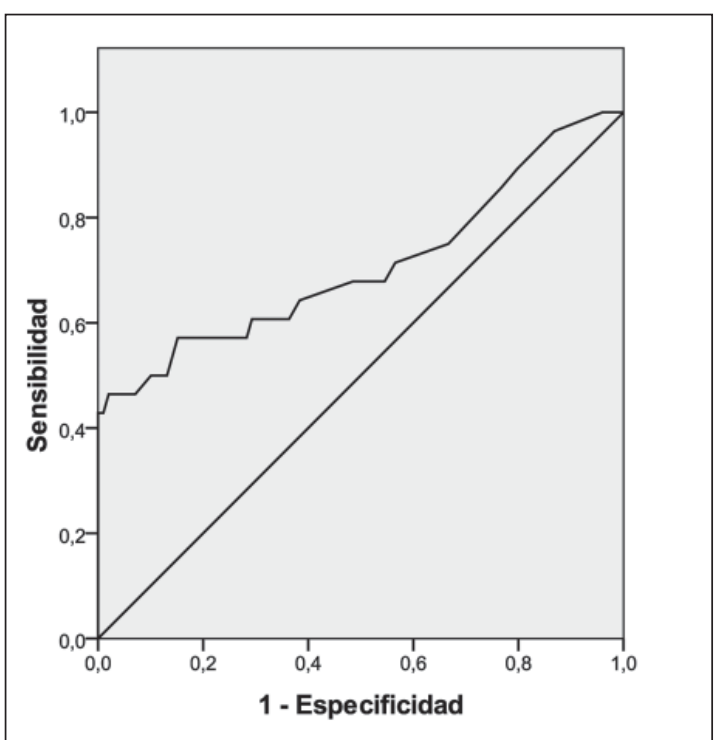

Figura 2. Curva ROC para los niveles séricos de APET en varones No Expuestos a concentraciones elevadas de As.

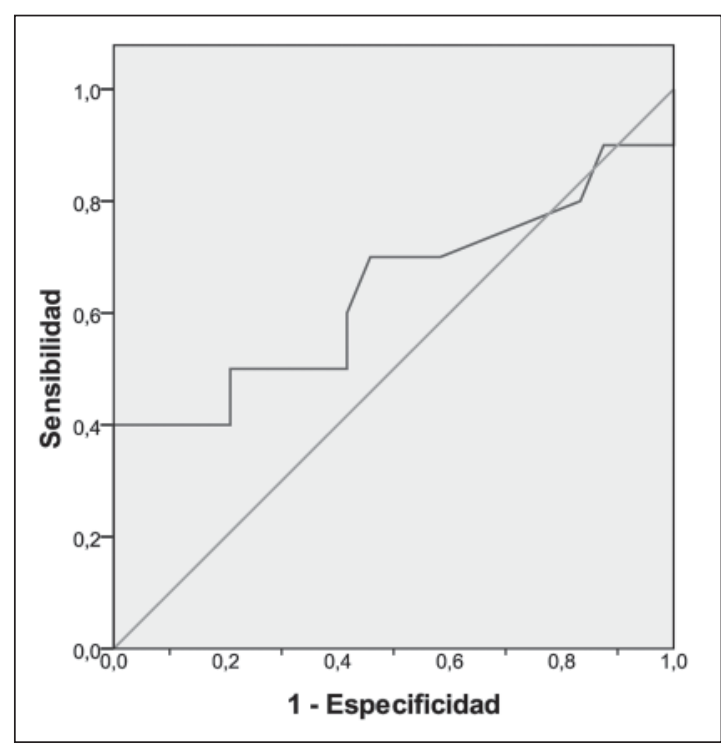

Figura 3. Curva ROC para los niveles séricos de APET en varones Expuestos a concentraciones elevadas de As. 


\section{Discusión}

En Argentina la mortalidad por todos los tumores representó, en el año 2004, 20\% de las causas de muerte, ocupando el segundo lugar. La mortalidad proporcional en varones de la Provincia de Tucumán corresponde en primer lugar a enfermedades del sistema circulatorio con 20,9\% seguido por los tumores malignos con 16,3\%. Las defunciones por tumores malignos en Tucumán corresponden en primer lugar al de CP con 135 muertes ${ }^{18}$. El arsenicismo crónico configura un grave problema de salud pública y alta importancia social ya que incide en comunidades pobres de la región este de la provincia de Tucumán ${ }^{12}$. El As ingerido en el agua de consumo es un agente carcinógeno, el cual es metabolizado y excretado por la orina como As, ácido monometilarsónico (MMA) y ácido dimetilarsínico (DMA). Un aumento del porcentaje de excreción del MMA es asociado con cáncer de piel, pulmón y vejiga en las personas expuestas ${ }^{19-21}$. El consumo de niveles elevados de As en el agua de bebida aumenta el riesgo de cáncer de vejiga, pulmón y riñón en hombres expuestos de Argentina y Chile ${ }^{16,22}$. En la población de Tucumán que consume agua contaminada con As, las lesiones en piel son las manifestaciones más comúnmente observadas. La hepatomegalia no es una manifestación frecuente $y$, en los pacientes que las presentaron, las alteraciones bioquímicas fueron compatibles con la presencia de un patrón colestásico ${ }^{23}$. En hombres que se encuentran expuestos y no expuestos al consumo de As en el agua de bebida la detección precoz de CP, puede acotar la severidad de este problema y el alto costo económico-social que produce. Por ello, en este trabajo se incluyeron varones asintomáticos expuestos y no expuestos a concentraciones elevadas de As en el agua de bebida, cuya distribución según los niveles del APET fue similar. Los porcentajes de $\mathrm{CP}$ en los grupos expuestos y no expuestos fueron de $3,0 \%$ y $3,1 \%$ respectivamente. Las primeras publicaciones en que se documentó la asociación entre mortalidad por CP y consumo de arsénico inorgánico, pertenecen a Chen y col en 1988 en Taiwán ${ }^{24}$. La mortalidad fue casi seis veces mayor que en la población general en el grupo expuesto a niveles altos de As en el agua de bebida (mayor o igual a 0,60 ppm). Cuando los niveles fueron estratificados ( $<0,30 \mathrm{ppm}, 0,30-0,59 \mathrm{ppm}$ y mayor o igual a $0,60 \mathrm{ppm}$ ) encontraron una relación dosis-respuesta significativa entre los niveles de As y la mortalidad por CP ajustada por la edad. En un estudio de cohorte realizado en una población de Estados Unidos de Norteamérica se encontró una mortalidad significativamente elevada de $\mathrm{CP}$ en la población expuesta al As en el agua de bebida $^{25}$. En otro estudio, en Australia, la relación de incidencia estandarizada en áreas con niveles elevados de As fue significativamente mayor para $\mathrm{CP}^{26}$. En este trabajo no se encontró evidencia de asociación entre exposición a niveles de As en el agua de consumo y $\mathrm{CP}$, probablemente porque el promedio de consumo de As en la población es baja $(0,16 \mathrm{mg} / \mathrm{mL})$ y el aumento de la mortalidad documentada por otros autores está en relación con la dosis consumida. El APET no fue capaz de diferenciar en los hombres con niveles menores o iguales a $4 \mathrm{ng} / \mathrm{mL}$ la presencia de patologías prostáticas entre los grupos expuestos y no expuestos. Cuando se analizaron los niveles séricos del APET en este grupo, se encontró que en la población expuesta las concentraciones fueron significativamente menores que en la población no expuesta. Existen evidencias experimentales que indican que el As puede causar efectos adversos sobre la función testicular, como pérdida de peso del testículo, disminución de la cantidad de esperma, y disminución de la actividad de $17 \beta$-hidroxiesteroide deshidrogenasa en ratones expuestos crónicamente a 4 ppm de As en el agua de bebida ${ }^{27}$. Como la secreción y concentración en sangre del APET es dependiente de los niveles de andrógenos, las diferencias observadas entre la población expuesta y no expuesta podrían explicarse por el efecto biológico del As sobre los niveles de la testosterona ${ }^{28}$. Para un valor de corte de $1,4 \mathrm{ng} / \mathrm{mL}$ la población no expuesta tiene una mejor sensibilidad (60\%) para diferenciar patologías prostáticas que la población expuesta (50\%), manteniendo en ambos grupos una especificidad similar. En una población de hombres entre 50 y 70 años, 8 a $9 \%$ tienen niveles de APET mayores o iguales a 4,0 $\mathrm{ng} / \mathrm{mL}, 11$ a $12 \%$ tienen valores mayores o iguales a $3 \mathrm{ng} / \mathrm{mL}$ y casi el $20 \%$ tienen valores mayores o iguales a 2,0 ng/mL 29 . El APET sólo no predice $\mathrm{CP}$, por lo tanto, no se debe indicar con este único parámetro la realización de biopsia prostática, ya que algunos cánceres diagnosticados son de bajo riesgo, no causando impacto en la expectativa de vida $^{30}$. El uso de los valores de corte determinados en este trabajo aumenta la sensibilidad para de- 
tectar $\mathrm{CP}$, sin embargo, aumentaría el número de biopsias innecesarias. Catalona y col demostraron que $20 \%$ de hombres con niveles entre 2 y $4 \mathrm{ng} / \mathrm{mL}$ tenían CP en la biopsia ${ }^{31}$. La prevalencia documentada de $\mathrm{CP}$ en hombres con edades comprendidas entre 62 y 91 años fue de 6,6\%,10\%,17\%,23,9\% y $26,9 \%$ con niveles de APET entre 0 y $0,5 \mathrm{mg} / \mathrm{mL}$, 0,6 y $1,0 \mathrm{mg} / \mathrm{mL}, 1,0$ y $2,0 \mathrm{mg} / \mathrm{mL}, 2,1$ y $3,0 \mathrm{mg} / \mathrm{mL}$ y 3,1 a $4,0 \mathrm{mg} / \mathrm{mL}$, respectivamente ${ }^{32}$. Aunque el tamaño de la muestra de nuestro estudio no nos permite plantear una conclusión definitiva, nos permite sugerir que el consumo crónico de As en el agua de bebida afecta la expresión del APET en los varones con niveles menores o iguales a 4 $\mathrm{ng} / \mathrm{mL}$, no encontrando evidencias de asociación entre la exposición al As y cáncer de próstata.Asimismo, es importante considerar en la evaluación urológica que la determinación del APET como método diagnóstico de $\mathrm{CP}$ en estadíos tempranos y potencialmente curable requiere de estudios complementarios, como el porcentaje del APE libre, el tacto rectal y la ecografía transrectal.

\section{Referencias}

1. Jemal A, Siegel R, Ward E, Hao Y, Xu J, Thun M. Cancer statistics, 2009. CA Cancer J Clin 2009; 59: 225-49.

2. Schmid HP, Engeler DS, Pummer K, Schmitz-Dräger BJ. Prevention of prostate cancer: more questions than data. Recent Results Cancer Res 2007; 174: 101-7.

3. Makinen T, Tammela TL, Hakama M, Stenman UH, Rannikko S, Aro J, et al. Tumor characteristics in a population-based prostate cancer screening trial with prostate-specific antigen. Clin Cancer Res 2003; 9: 24359.

4. Draisma G, Boer R, Otto SJ, van der Cruijsen IW, Damhuis RA, Schroder FH, et al. Lead times and overdetection due to prostate-specific antigen screening: estimates from the European Randomized Study of Screening for Prostate Cancer. J Natl Cancer Inst 2003; 95: 868-78.

5. Nadler RB, Collins MM, Propert KJ, Mikolajczyk SD, Knauss JS, Landis JR, et al. Chronic Prostatitis Collaborative Research Network Prostate-specific antigen test in diagnostic evaluation of chronic prostatitis/chronic pelvic pain syndrome. Urology 2006; 67: 337-42.

6. Wolf A, Wender RC, Etzioni RB, Thompson IM, D'Amico AV, Volk RJ, et al. American Cancer Society Guideline for the Early Detection of Prostate Cancer. Update 2010. CA Cancer J Clin 2010; 60: 70-98.

7. Crawford ED, Leewansangtong S, Goktas S, Holthaus $\mathrm{K}$, Baier M. Efficiency of prostate-specific antigen and digital rectal examination in screening, using $4.0 \mathrm{ng} / \mathrm{ml}$ and age-specific reference range as a cutoff for abnormal values. Prostate 1999; 38: 296-302.

8. Roddam AW, Duffy MJ, Hamdy FC, Ward AM, Patnick J, Price CP, et al. Use of prostate-specific antigen (PSA) isoforms for the detection of prostate cancer in men with a PSA level of 2-10 ng/ml: systematic review and metaanalysis. Eur Urol 2005; 48: 386-98; discussion 398-9.

9. Catalona WJ, Partin AW, Slawin KM, Brawer MK, Flanigan RC, Patel A, et al. Use of the percentage of free prostate-specific antigen to enhance differentiation of prostate cancer from benign prostatic disease: a prospective multicenter clinical trial. JAMA 1998; 279: 1542-7.

10. IARC Working Group on the Evaluation of Carcinogenic Risks to Humans. Some drinking-water disinfectants and contaminants, including arsenic. IARC Monogr Eval Carcinog Risks Hum 2004; 84: 269-477.

11. Benbrahim-Tallaa L, Waalkes MP. Inorganic arsenic and human prostate cancer. Environ. Health Perspect 2008; 116: 158-64.

12. Guber RS, Tefaha L, Arias NN, Sandoval NG, Toledo R, Fernandez M, et al. Contenido de arsénico en el agua de consumo en Leales y Graneros (Provincia de TucumánArgentina). Acta Bioquím Clín Latinoam 2009; 43: 2017.

13. U.S. EPA. Arsenic, Inorganic (CASRN 7440-38-2), Integrated Risk Information System. Washington, DC: U.S. Environmental Protection Agency 1993. Available: http://www.epa.gov/iris/subst/0278.htm [31 March 2005].

14. International Agency for Research on Cancer. Arsenic and arsenic compounds. IARC Monogr Eval Carcinog Risks Hum 1987; 23: 100-3.

15. Tokar EJ, Qu W, Liu J, Liu W, Webber MM, Phang JM, Waalkes MP. Arsenic-specific stem cell selection during malignant transformation. J Natl Cancer Inst 2010; 102: 638-49.

16. Rivara MI, Corey G. Tendency to die of cancers associated to chronic exposure to arsenic, II Region of Antofagasta, 1950-1993. Cuad Méd Soc (Chile) 1995; 36: 39-51.

17. Smith AH, Marshall G, Yuan Y, Ferreccio C, Liaw J, von Ehrenstein O, et al. Increased Mortality from Lung Cancer and Bronchiectasis in Young Adults Following Exposure to Arsenic In Utero and Early Childhood. Environ Health Perspect 2006; 114: 1293-6.

18. Ministerio de Salud de la Nación. Secretaría de Políticas, Regulación e Institutos. Dirección de Estadísticas e Información de salud. Estadísticas vitales. Información básica-2008. Serie 5, Número 52, 2009. Disponible en http://www.deis.gov.ar/Publicaciones/Archivos/Serie5Nro52.pdf. [Consultado el 15 de octubre de 2010]. 
19. Huang YK, Huang YL, Hsueh YM, Yang MH, Wu MM, Chen SY, et al. Arsenic exposure, urinary arsenic speciation, and the incidence of urothelial carcinoma: a twelve-year follow-up study. Cancer Causes Control 2008; 19: 829-39.

20. Tseng $\mathrm{CH}$. Arsenic methylation, urinary arsenic metabolites and human diseases: current perspective. J Environ Sci Health C Environ Carcinog Ecotoxicol Rev 2007; 25: $1-22$.

21. Steinmaus C, Yuan Y, Kalman D, Rey OA, Skibola CF, Dauphine $\mathrm{D}$, et al. Individual differences in arsenic metabolism and lung cancer in a case-control study in Cordoba, Argentina. Toxicol Appl Pharmacol 2010; 247: 138-45.

22. Hopenhayn-Rich C, Biggs Smith H. Lung and kidney cancer mortality associated with arsenic in drinking water in Cordoba, Argentina. Int J Epidemiol 1998; 27: 561-9.

23. Soria de González A, Guber R, Martínez M, Arias N, Tefaha L, Sandoval N, et al. Alteraciones bioquímicas en individuos expuestos al arsénico en el agua de bebida en Tucumán, Argentina. Acta Bioquím Clín Latinoam 2009; 43: 611-8.

24. Chen CJ, Kuo TL, Wu MM. Arsenic and cancers. Lancet 1988; 1: 414-5.

25. Lewis DR, Southwick JW, Ouellet-Hellstrom R, Rench J, Calderon RL. Drinking water arsenic in Utah: a cohort mortality study. Environ Health Perspect 1999; 107: 35965.
26. Hinwood AL, Jolley DJ, Sim MR. Cancer incidence and high environmental arsenic concentrations in rural populations: results of an ecological study. Int J Environ Health R 1999; 9: 131-41.

27. Pant N, Murthy RC, Srivastava SP. Male reproductive toxicity of sodium arsenite in mice. Hum Exp Toxicol 2004; 23: 399-403.

28. Hsieh FI, Hwang TS, Hsieh YCh, Lo HCh, Su ChT, Hsu HS, et al. Risk of Erectile Dysfunction Induced by Arsenic Exposure through Well Water Consumption in Taiwan. Environ Health Perspect 2008; 116: 532-36.

29. Aus G, Damber JE, Khatami A, Lilja H, Stranne J, Hugosson J. Individualized screening interval for prostate cancer based on prostate-specific antigen level: results of a prospective, randomized, population-based study. Arch Intern Med 2005; 165: 1857-61.

30. Graham J, Baker M, Macbeth F, Titshall V. Diagnosis and treatment of prostate cancer: summary of NICE guidance. BMJ 2008; 336: 610-2.

31. Catalona WJ, Smith DS, Ornstein DK. Prostate cancer detection in men with serum PSA concentrations of 2.6 to $4.0 \mathrm{ng} / \mathrm{mL}$ and benign prostate examination: enhancement of specificity with free PSA measurements. JAMA 1997; 277: 1452-5.

32. Thompson IM, Pauler DK, Goodman PJ, Tangen CM, Lucia MS, Parnes HL, et al. Prevalence of Prostate Cancer among Men with a Prostate-Specific Antigen Level $\leq 4.0$ ng per Milliliter. N Engl J Med 2004; 350: 2239-46. 